\title{
Attractors for parabolic equations related to Caffarelli-Kohn-Nirenberg inequalities
}

\author{
Nguyen Dinh Binh ${ }^{1 *}$ and Cung The $\mathrm{Anh}^{2}$
}

\author{
* Correspondence: binhngd- \\ fami@mail.hut.edu.vn \\ 'Department of Applied \\ Mathematics and Informatics, \\ Hanoi University of Science and \\ Technology, 1 Dai Co Viet, Hai Ba \\ Trung, Hanoi, Vietnam \\ Full list of author information is \\ available at the end of the article
}

\begin{abstract}
Using the theory of uniform global attractors for multi-valued semiprocesses, we prove the existence of attractors for quasilinear parabolic equations related to Caffarelli-Kohn- Nirenberg inequalities, in which the conditions imposed on the nonlinearity provide the global existence of weak solutions but not uniqueness, in both autonomous and non-autonomous cases.

Mathematics Subject Classification 2010: 35B41, 35K65, 35D30.

Keywords: Caffarelli-Kohn-Nirenberg inequalities, non-uniqueness, weak solution, multivalued semiflow, multi-valued semiprocess, compact attractor, compactness and monotonicity methods
\end{abstract}

\section{Introduction}

The understanding of the asymptotic behavior of dynamical systems is one of the most important problems of modern mathematical physics. One way to attack the problem for a dissipative dynamical system is to consider its attractor. The existence of the attractor has been derived for a large class of PDEs (see e.g., [1,2] and references therein) for both autonomous and non-autonomous equations. However, these researches may not be applied to a wide class of problems, in which solutions may not be unique. Good examples of such systems are differential inclusions, variational inequalities, control infinite dimensional systems and also some partial differential equations for which solutions may not be known unique as, for example, some certain semilinear wave equations with high power nonlinearities, the incompressible NavierStokes equation in three space dimension, the Ginzburg-Landau equation, etc. For the qualitative analysis of the above mentioned systems from the point of view of the theory of dynamical systems, it is necessary to develop a corresponding theory for multivalued semigroups.

In the last years, there have been some theories for which one can treat multi-valued semi-flows and their asymptotic behavior, including the generalized semiflows theory of Ball [3], theory of trajectory attractors of Chepyzhov and Vishik [4] and theories of multi-valued semiflows and semiprocesses of Melnik and Valero [5-7]. Thanks to these theories, several results concerning attractors in the case of equations without uniqueness have been obtained recently for differential inclusion [5,6], parabolic equations [8-10], the phase-field equation [11], the wave equation [12], the three-dimensional Navier-Stokes equation $[3,13]$, etc. Although the existence of attractors has been

(c) 2012 Binh and Anh; licensee Springer. This is an Open Access article distributed under the terms of the Creative Commons Attribution License (http://creativecommons.org/licenses/by/2.0), which permits unrestricted use, distribution, and reproduction in any medium, provided the original work is properly cited. 
derived for many classes of partial differential equations without uniqueness, to the best of our knowledge, little seems to be known for singular/degenerate equations, expecially in the quasilinear case.

Let $\Omega$ be a bounded domain in $\mathbb{R}^{N}(N \geq 2)$ containing the origin with boundary $\partial \Omega$. In this paper we consider the following quasilinear parabolic equation

$$
\begin{aligned}
\frac{\partial u}{\partial t}-\operatorname{div}\left(|x|^{-p \gamma}|\nabla u|^{p-2} \nabla u\right)+ & f(t, u)=g(x, t), \quad x \in \Omega, \quad t>\tau, \\
\left.u\right|_{t=\tau} & =u_{\tau}(x), \quad x \in \Omega, \\
\left.u\right|_{\partial \Omega} & =0,
\end{aligned}
$$

where $\tau \in \mathbb{R}, u_{\tau} \in L^{2}(\Omega)$ are given, the nonlinearity $f$, the external force $g$, and the numbers $p, \gamma$ satisfy the following conditions:

(H1) $f: \mathbb{R} \times \mathbb{R} \rightarrow \mathbb{R}$ is a continuous function satisfying

$$
\begin{aligned}
& |f(t, u)| \leq C_{1}|u|^{q-1}+k_{1}, \\
& u f(t, u) \geq C_{2}|u|^{q}-k_{2},
\end{aligned}
$$

for some $q \geq 2$, where $C_{1}, C_{2}, k_{1}, k_{2}$ are positive constants;

(H2) $g \in L_{c}^{2}\left(\mathbb{R} ; L^{2}(\Omega)\right)$, where $L_{c}^{2}\left(\mathbb{R} ; L^{2}(\Omega)\right)$ is the set of all translation compact functions in $L_{\text {loc }}^{2}\left(\mathbb{R} ; L^{2}(\Omega)\right)$ whose definition is given in Definition 1.1 below.

(H3) $\frac{2 N}{N+2} \leq p \leq 2$ and $\frac{N}{p}-\frac{N}{2} \leq \gamma+1<\frac{N}{p}$.

Let us give some comments about assumptions $(H 1)-(H 3)$. The nonlinearity $f$ is assumed to have a polynomial growth and to satisfy a standard dissipative condition. A typical example of functions satisfying conditions $(H 1)$ is $f(t, u)=|u|^{q-2} u$. $\arctan t, q \geq 2$. We refer the reader to [[1], Chapter 5, Propositions 3.3 and 3.5] for translation compact criterions in $L_{\text {loc }}^{2}\left(\mathbb{R} ; L^{2}(\Omega)\right)$. While $(H 3)$ is a technical condition ensuring that $\mathcal{D}_{0, \gamma}^{1, p}(\Omega)$ is embedded compactly into $L^{2}(\Omega)$, where $\mathcal{D}_{0, \gamma}^{1, p}(\Omega)$ is the natural energy space related to problem (1.1), which is defined later in this section. This is essential for proving the existence of a weak solution to problem (1.1) using the compactness method.

Problem (1.1), which is related to some Caffarelli-Kohn-Nirenberg inequalities [14], contains some important classes of parabolic equations, such as the semilinear heat equations (when $\gamma=0, p=2$ ), semilinear singular/degenerate parabolic equations (when $p=2$ ), the $p$-Laplacian equations (when $\gamma=0, p \neq 2$ ), etc. The existence and properties of solutions to problem type (1.1) have attracted interest in recent years [15-19]. However, to the best of our knowledge, little seems to be known for the longtime behavior of solutions to problem (1.1).

In this article we study the long-time behavior of solutions to problem (1.1) via the concept of uniform global attractors for multi-valued semiprocesses. Here there is no restrictions on the growth of the nonlinearity $f$ and the conditions imposed on $f$ provide the global existence of a weak solution to problem (1.1), but not uniqueness. Thus, when studying the long-time behavior of solutions, in order to handle 
nonuniqueness of solutions, we need use the theory of attractors for multi-valued semiprocesses. Following the general lines of the approach used in $[8-10,20]$ for nondegenerate parabolic equations, we prove the existence of a global compact attractor in the autonomous case, and of a uniform global compact attractor in the non-autonomous case. Noting that when the nonlinearity $f$ does not depend on time $t$, the existence of an attractor for problem (1.1) in the semilinear non-degenerate case, namely when $\gamma=0$ and $p=2$, was studied in [8,9]. Thus, our results extend some known results on the existence and long-time behavior of solutions of nondegenerate semilinear parabolic equations.

It is worth noticing that under some additional conditions on $f$, for example, $f_{u}^{\prime}(t, u) \geq-C_{3}$ for all $t>\tau, u \in \mathbb{R}$, or a weaker assumption

$$
(f(t, u)-f(t, v))(u-v) \geq-C|u-v|^{2} \text { for all } t>\tau, u, v \in \mathbb{R},
$$

one can prove that the weak solution of problem (1.1) is unique. Then the multivalued semiprocess turns to be a single-valued one and the uniform compact global attractor is exactly the usual uniform attractor for the family of single-valued semiprocesses [1].

In the rest of this section, for convenience of the reader, we recall some results on function spaces related to Caffarelli-Kohn-Nirenberg inequalities and translation compact functions.

For $1<p<\infty$ and $\gamma<\frac{N-p}{p}$, we define the weighted space

$$
L_{\gamma}^{p}(\Omega)=\left\{u: \Omega \rightarrow \mathbb{R} \text { is measurable such that }|x|^{-\gamma} u(x) \in L^{p}(\Omega)\right\},
$$

equipped with the norm

$$
\|u\|_{L_{\gamma}^{p}(\Omega)}=\left(\int_{\Omega}|x|^{-p \gamma}|u(x)|^{p}\right)^{1 / p} .
$$

It is easy to check that the dual space $\left(L_{\gamma}^{p}(\Omega)\right)^{\prime}$ of $L_{\gamma}^{p}(\Omega)$ is the space $L_{-\gamma}^{p^{\prime}}(\Omega)$, where $p^{\prime}$ is defined by $\frac{1}{p}+\frac{1}{p^{\prime}}=1$. Moreover, we define the weighted Sobolev space $\mathcal{D}_{0, \gamma}^{1, p}(\Omega)$ as the closure of $C_{0}^{\infty}(\Omega)$ in the norm

$$
\|u\|_{\mathcal{D}_{0, \gamma}^{1, p}(\Omega)}=\|\nabla u\|_{L_{\gamma}^{p}(\Omega)}=\left(\int_{\Omega}|x|^{-p \gamma}|\nabla u(x)|^{p} d x\right)^{\frac{1}{p}} .
$$

As $1<p<\infty, \mathcal{D}_{0, \gamma}^{1, p}(\Omega)$ is reflexive, and the dual space of $\mathcal{D}_{0, \gamma}^{1, p}(\Omega)$ will be denoted by $\mathcal{D}_{-\gamma}^{-1, p^{\prime}}(\Omega)$

We now state some results which we will use later. The first is the Caffarelli-KohnNirenberg inequality.

Proposition 1.1. [14]Assume that $1<p<N$. Then there exists a positive constant $C_{N, p \text {, }}$ r,q such that for every $u \in C_{0}^{\infty}\left(\mathbb{R}^{N}\right)$, 


$$
\left(\int_{\mathbb{R}^{N}}|x|^{-\delta q}|u(x)|^{q} d x\right)^{p / q} \leq C_{N, p, \gamma, q} \int_{\mathbb{R}^{N}}|x|^{-p \gamma}|\nabla u(x)|^{p} d x
$$

where $p, q, \gamma, \delta$ are related by

$$
\frac{1}{q}-\frac{\delta}{N}=\frac{1}{p}-\frac{\gamma+1}{N}, \quad \gamma \leq \delta \leq \gamma+1,
$$

and $\delta q<N, \gamma p<N$.

The inequality (1.5) implies that the embedding

$$
\mathcal{D}_{0, \gamma}^{1, p}(\Omega) \subset L_{\delta}^{q}(\Omega) \text { is continuous for } p, q, \gamma, \delta \text { satisfying (1.6). }
$$

This implies, by duality,

$$
L_{-\delta}^{q^{\prime}}(\Omega) \subset \mathcal{D}_{-\gamma}^{-1, p^{\prime}}(\Omega) \text { for } p, q, \gamma, \delta \text { satisfying (1.6). }
$$

It is pointed out in [19] that

$$
\mathcal{D}_{0, \gamma}^{1, p}(\Omega) \subset L_{\delta}^{q}(\Omega) \text { compactly }
$$

for every $p, q, \gamma, \delta$ satisfying $\frac{1}{q}-\frac{\delta}{N}>\frac{1}{p}-\frac{\gamma+1}{N}$ with $\gamma \leq \delta \leq \gamma+1$ and $\delta q<N, \gamma p$ $<N$.

From assumption (H3), it is easy to check that there exists a positive number $\delta$ such that $\mathcal{D}_{0, \gamma}^{1, p}(\Omega) \subset L_{\delta}^{2}(\Omega)$ compactly. Since the embedding $L_{\delta}^{2}(\Omega) \subset L^{2}(\Omega)$ is continuous, it is seen that $\mathcal{D}_{0, \gamma}^{1, p}(\Omega) \subset \subset L^{2}(\Omega) \subset \mathcal{D}_{-\gamma}^{-1, p^{\prime}}(\Omega)$ ) is an evolution triplet.

We now define the following "evolution" spaces which will be useful in what follows.

$$
\begin{aligned}
L^{p}\left(\tau, T ; \mathcal{D}_{0, \gamma}^{1, p}(\Omega)\right)= & \{u(., .): \Omega \times(\tau, T) \rightarrow \mathbb{R} \text { measurable: } \\
& \left.u(., t) \in \mathcal{D}_{0, \gamma}^{1, p}(\Omega) \quad \text { for a.e. } t \in(\tau, T),\|u(., t)\|_{\mathcal{D}_{0, \gamma}^{1, p}(\Omega)} \in L^{p}(\tau, T)\right\},
\end{aligned}
$$

endowed with the norm

$$
\|u\|_{L^{p}\left(\tau, T ; \mathcal{D}_{0, \gamma}^{1, p}(\Omega)\right)}=\left(\int_{\tau}^{T}\|u(., t)\|_{\mathcal{D}_{0, \gamma}^{1, p}(\Omega)}^{p} d t\right)^{1 / p}=\left(\int_{\tau}^{T} \int_{\Omega}^{T}|x|^{-p \gamma}|\nabla u|^{p} d x d t\right)^{1 / p} .
$$

The dual space of $L^{p}\left(\tau, T ; \mathcal{D}_{0, \gamma}^{1, p}(\Omega)\right)$ is $L^{p^{\prime}}\left(\tau, T ; \mathcal{D}_{-\gamma}^{-1, p^{\prime}}(\Omega)\right)$.

Putting

$$
-\Delta_{p, \gamma} u=-\operatorname{div}\left(|x|^{-p \gamma}|\nabla u|^{p-2} \nabla u\right), \quad u \in \mathcal{D}_{0, \gamma}^{1, p}(\Omega)
$$

The following proposition, which is easily proved by using similar arguments as in [[21], Chapter 2], gives some important properties of the operator $-\Delta_{p, \gamma}$

Proposition 1.2. The operator $-\Delta_{p, \gamma}$ maps $\mathcal{D}_{0, \gamma}^{1, p}(\Omega)$ into its dual $\mathcal{D}_{-\gamma}^{-1, p^{\prime}}(\Omega)$. Moreover, 
(1) $-\Delta_{p, \gamma}$ is hemicontinuous, i.e., for all $u, v, w \in \mathcal{D}_{0, \gamma}^{1, p}(\Omega)$, the map $\lambda \mapsto\left\langle-\Delta_{p, \gamma}(u+\right.$ $\lambda v)$, w $>$ is continuous from $\mathbb{R}$ to $\mathbb{R}$.

(2) $-\Delta_{p, \gamma}$ is monotone, i.e., $\left\langle-\Delta_{p, \gamma} u+\Delta_{p, \gamma} v, u-v\right\rangle \geq 0$, for all $u, v \in \mathcal{D}_{0, \gamma}^{1, p}(\Omega)$.

Definition 1.1. Assume that $\mathscr{E}$ is a reflexive Banach space.

(1) A function $\varphi \in L_{\mathrm{loc}}^{2}(\mathbb{R} ; \mathcal{E})$ is said to be translation bounded if

$$
\|\varphi\|_{L_{b}^{2}}^{2}=\|\varphi\|_{L_{b}^{2}(\mathbb{R} ; \mathcal{E})}=\sup _{t \in \mathbb{R}} \int_{t}^{t+1}\|\varphi\|_{\mathcal{E}}^{2} d s<\infty .
$$

(2) A function $\varphi \in L_{\text {loc }}^{2}(\mathbb{R} ; \mathcal{E})$ is said to be translation compact if the closure of $\{\phi(\cdot$

$+h) \mid h \in \mathbb{R}\}$ is compact in $L_{\mathrm{loc}}^{2}(\mathbb{R} ; \mathcal{E})$.

Denote by $L_{b}^{2}(\mathbb{R} ; \mathcal{E})$ and $L_{c}^{2}(\mathbb{R} ; \mathcal{E})$ the sets of all translation bounded functions and of all translation compact functions in $L_{\text {loc }}^{2}(\mathbb{R} ; \mathcal{E})$, respectively. It is well-known (see [4]) that $L_{c}^{2}(\mathbb{R} ; \mathcal{E}) \subset L_{b}^{2}(\mathbb{R} ; \mathcal{E})$.

Let $\mathcal{H}(g)$ be the closure of the set $\{g(\cdot+h) \mid h \in \mathbb{R}\}$ in $L_{b}^{2}\left(\mathbb{R} ; L^{2}(\Omega)\right)$. The following results were proved in [[1], Chapter 5, Proposition 3.4].

Lemma 1.3. (1) $\mathcal{H}(g)$ is compact.

(2) For all $\sigma \in \mathcal{H}(g),\|\sigma\|_{L_{b}^{2}}^{2} \leq\|g\|_{L_{b}^{2}}^{2}$;

(3) The translation group $\{T(h)\}$, which is defined by $T(h) \sigma(s)=\sigma(h+s), s, h \in \mathbb{R}$, is continuous on $\mathcal{H}(\mathrm{g})$;

(4) $T(h) \mathcal{H}(g)=\mathcal{H}(g)$ for $h \geq 0$;

The rest of the article is organized as follows. In Section 2, we prove the global existence of a weak solution to problem (1.1) by using the monotonicity and compactness methods. In Section 3, the existence of global attractors for problem (1.1) is proved in both the autonomous and non-autonomous cases.

\section{Existence of a weak solution}

We denote

$$
\begin{aligned}
Q_{\tau, T} & =\Omega \times(\tau, T), \\
V & =L^{p}\left(\tau, T ; \mathcal{D}_{0, \gamma}^{1, p}(\Omega)\right) \cap L^{q}\left(\tau, T ; L^{q}(\Omega)\right), \\
V^{\prime} & =L^{p^{\prime}}\left(\tau, T ; \mathcal{D}_{-\gamma}^{-1, p^{\prime}}(\Omega)\right)+L^{q^{\prime}}\left(\tau, T ; L^{q^{\prime}}(\Omega)\right),
\end{aligned}
$$

where $p^{\prime}, q^{\prime}$ are the conjugate indexes of $p, q$, respectively. 
Definition 2.1. A function $u(x, t)$ is called a weak solution of $(1.1)$ on $(\tau, T)$ iff

$$
\begin{gathered}
u \in V, \quad \frac{d u}{d t} \in V^{\prime}, \\
\left.u\right|_{t=\tau}=u_{\tau} \quad \text { a.e. in } \Omega,
\end{gathered}
$$

and

$$
\int_{\tau}^{T}\left\langle u_{t}, \varphi\right\rangle d t+\int_{\tau}^{T} \int_{\Omega}|x|^{-p \gamma}|\nabla u|^{p-2} \mid \nabla u \nabla \varphi d x d t+\int_{\tau}^{T}\langle f(t, u), \varphi\rangle d t=\int_{\tau}^{T}(g(t), \varphi) d t
$$

for all test functions $\phi \in V$.

It is known (see [[1], Theorem 1.8, p. 33]) that if $u \in V$ and $\frac{d u}{d t} \in V^{\prime}$, then $u \in C([\tau$, $T] ; L^{2}(\Omega)$ ). This makes the initial condition in problem (1.1) meaningful.

Theorem 2.1. For any $\tau, T \in \mathbb{R}, T>\tau$ and $u_{\tau} \in L^{2}(\Omega)$ given, problem (1.1) has at least one weak solution $u$ on $(\tau, T)$. Moreover, the solution $u$ can be extended to the whole interval $(\tau,+\infty)$.

Proof. We split the proof into three steps.

Step 1: A Galerkin scheme. Consider the approximating solution $u_{n}(t)$ in the form

$$
u_{n}(t)=\sum_{k=1}^{n} u_{n k}(t) e_{k}
$$

where $\left\{e_{k}\right\}_{k=1}^{\infty}$ is a basis of $\left.\mathcal{D}_{0, \gamma}^{1, p}(\Omega)\right) \cap L^{q}(\Omega)$, which is orthonormal in $L^{2}(\Omega)$. We get $u_{n}$ from solving the problem

$$
\begin{aligned}
& \left\langle\frac{d u_{n}}{d t}, e_{k}\right\rangle+\left\langle-\Delta_{p, \gamma} u_{n}, e_{k}\right\rangle+\left\langle f\left(t, u_{n}\right), e_{k}\right\rangle=\left(g(t), e_{k}\right), \\
& \left(u_{n}(\tau), e_{k}\right)=\left(u_{\tau}, e_{k}\right), \quad k=1, \ldots, n .
\end{aligned}
$$

Using the Peano theorem in the theory of ODEs, we get the local existence of $u_{n}$.

Step 2: A priori estimates. We have

$$
\frac{1}{2} \frac{d}{d t}\left\|u_{n}\right\|_{L^{2}(\Omega)}^{2}+\left\|u_{n}\right\|_{\mathcal{D}_{0, \gamma}^{1, p}(\Omega)}^{p}+\int_{\Omega} f\left(t, u_{n}\right) u_{n} d x=\int_{\Omega} g(t) u_{n} d x .
$$

By assumption (H3), we can choose $\delta>0$ such that $\frac{1}{2}-\frac{\delta}{N}>\frac{1}{p}-\frac{\gamma+1}{N}$, then $\mathcal{D}_{0, \gamma}^{1, p}(\Omega) \subset \subset L_{\delta}^{2}(\Omega) \subset L^{2}(\Omega)$ and therefore there exists $\lambda>0$ such that

$$
\|u\|_{\mathcal{D}_{0, \gamma}^{1, p}(\Omega)}^{p} \geq C\|u\|_{L_{\delta}^{2}(\Omega)}^{p} \geq \hat{\lambda}\|u\|_{L^{2}(\Omega)}^{p} \geq \lambda\|u\|_{L^{2}(\Omega)}^{2}-\lambda,
$$

where the last inequality follows from the Young inequality. Using (1.3) and the Cauchy inequality, we get

$$
\frac{1}{2} \frac{d}{d t}\left\|u_{n}\right\|_{L^{2}(\Omega)}^{2}+\left\|u_{n}\right\|_{\mathcal{D}_{0, \gamma}^{1, p}(\Omega)}^{p}+C_{2}\left\|u_{n}\right\|_{L^{q}(\Omega)}^{q}-k_{2}|\Omega| \leq \frac{1}{2 \lambda}\|g(t)\|_{L^{2}(\Omega)}^{2}+\frac{\lambda}{2}\left\|u_{n}\right\|_{L^{2}(\Omega)}^{2} .
$$


Hence

$$
\frac{d}{d t}\left\|u_{n}\right\|_{L^{2}(\Omega)}^{2}+\left\|u_{n}\right\|_{\mathcal{D}_{0, \gamma}^{1, p}(\Omega)}^{p}+2 C_{2}\left\|u_{n}\right\|_{L^{q}(\Omega)}^{q} \leq \frac{1}{\lambda}\|g(t)\|_{L^{2}(\Omega)}^{2}+2 k_{2}|\Omega|+\lambda .
$$

We show that the local solution $u_{n}$ can be extended to the interval $[\tau, \infty)$. Indeed, from (2.2) we have

$$
\frac{d}{d t}\left\|u_{n}\right\|_{L^{2}(\Omega)}^{2}+\lambda\left\|u_{n}\right\|_{L^{2}(\Omega)}^{2} \leq \frac{1}{\lambda}\|g(t)\|_{L^{2}(\Omega)}^{2}+2 k_{2}|\Omega|+2 \lambda
$$

By the Gronwall inequality, we obtain

$$
\begin{aligned}
\left\|u_{n}(t)\right\|_{L^{2}(\Omega)}^{2} \leq & \left\|u_{n}(\tau)\right\|_{L^{2}(\Omega)}^{2} e^{-\lambda(t-\tau)}+\frac{1}{\lambda} \int_{\tau}^{t} e^{-\lambda(t-s)}\|g(s)\|_{L^{2} \Omega}^{2} d s \\
& +\left(2 k_{2}|\Omega|+2 \lambda\right) \int_{\tau}^{t} e^{-\lambda(t-s)} d s \\
\leq & \left\|u_{\tau}\right\|_{L^{2}(\Omega)}^{2} e^{-\lambda(t-\tau)}+\frac{1}{\lambda\left(1-e^{-\lambda}\right)}\|g\|_{L_{b}^{2}}^{2}+\frac{2 k_{2}|\Omega|}{\lambda}+2,
\end{aligned}
$$

where we have used the facts that $\left\|u_{n}(\tau)\right\|_{L^{2}(\Omega)} \leq\left\|u_{\tau}\right\|_{L^{2}(\Omega)}$ and

$$
\begin{aligned}
\int_{\tau}^{t} e^{-\lambda(t-s)}\|g(s)\|_{L^{2}(\Omega)}^{2} d s & \leq \int_{t-1}^{t} e^{-\lambda(t-s)}\|g(s)\|_{L^{2}(\Omega)}^{2} d s+\int_{t-2}^{t-1} e^{-\lambda(t-s)}\|g(s)\|_{L^{2}(\Omega)}^{2} d s+\cdots \\
& \leq \int_{t-1}^{t}\|g(s)\|_{L^{2}(\Omega)}^{2} d s+e^{-\lambda} \int_{t-2}^{t-1}\|g(s)\|_{L^{2}(\Omega)}^{2} d s+\cdots \\
& \leq\left(1+e^{-\lambda}+e^{-2 \lambda}+\cdots\right)\|g\|_{L_{b}^{2}}^{2}=\frac{1}{1-e^{-\lambda}}\|g\|_{L_{b}^{2}}^{2}
\end{aligned}
$$

We now establish some a priori estimates for $u_{n}$. Integrating (2.2) on $[\tau, T], \tau<t \leq T$, and using the fact that $\left\|u_{n}(\tau)\right\|_{L^{2}(\Omega)} \leq\left\|u_{\tau}\right\|_{L^{2}(\Omega)}$, we have

$$
\begin{aligned}
\left\|u_{n}(t)\right\|_{L^{2}(\Omega)}^{2} & +\int_{\tau}^{T}\left\|u_{n}(s)\right\|_{\mathcal{D}_{0, \gamma}^{1, p}(\Omega)}^{p} d s+2 C_{2} \int_{\tau}^{T}\left\|u_{n}(s)\right\|_{L^{q}(\Omega)}^{q} d s \\
& \leq\left\|u_{\tau}\right\|_{L^{2}(\Omega)}^{2}+\frac{1}{\lambda} \int_{\tau}^{T}\|g(s)\|_{L^{2} \Omega}^{2} d s+\left(2 k_{2}|\Omega|+2 \lambda\right)(T-\tau) .
\end{aligned}
$$

The last inequality implies that

$\left\{u_{n}\right\}$ is bounded in $L^{\infty}\left(\tau, T ; L^{2}(\Omega)\right)$,

$\left\{u_{n}\right\}$ is bounded in $L^{p}\left(\tau, T ; \mathcal{D}_{0, \gamma}^{1, p}(\Omega)\right)$,

$\left\{u_{n}\right\}$ is bounded in $L^{q}\left(\tau, T ; L^{q}(\Omega)\right)$. 
Using hypothesis (1.2), we get

$$
\int_{\tau}^{T}\left\|f\left(t, u_{n}\right)\right\|_{L^{\prime \prime}(\Omega)}^{q \prime^{\prime}} d t \leq \int_{\tau}^{T} \int_{\Omega}^{T}\left(C_{1}\left|u_{n}\right|^{q-1}+k_{1}\right)^{q \prime} d x d t \leq \int_{\tau}^{T} \int_{\Omega} C\left(\left|u_{n}\right|^{q}+1\right) d x d t .
$$

Hence, we can conclude that $\left\{f\left(t, u_{n}\right)\right\}$ is bounded in $L^{q^{\prime}}\left(\tau, T ; L^{q^{\prime}}(\Omega)\right)$ and thus,

$$
f\left(t, u_{n}\right) \rightarrow \eta \text { in } L^{q^{\prime}}\left(\tau, T ; L^{q^{\prime}}(\Omega)\right) .
$$

We have

$$
\begin{aligned}
\left|\left\langle-\Delta_{p, \gamma} u_{n}, v\right\rangle\right| & =\left|\left\langle-\operatorname{div}\left(|x|^{-p \gamma}|\nabla u|^{p-2} \nabla u\right), v\right\rangle\right| \\
& =\left.\left|\int_{\tau}^{T} \int_{\Omega}\right| x\right|^{-p \gamma}\left|\nabla u_{n}\right|^{p-2} \nabla u_{n} \nabla v d x d t \mid \\
& =\left.\left|\int_{\tau}^{T} \int_{\Omega}\right| x\right|^{-(p-1) \gamma}\left|\nabla u_{n}\right|^{p-2} \nabla u_{n}\left(|x|^{-\gamma} \nabla v\right) d x d t \mid \\
& \leq\left\|u_{n}\right\|_{L^{p}\left(\tau, T ; \mathcal{D}_{0, \gamma}^{1, p}(\Omega)\right)}\|v\|_{L^{p}\left(\tau, T ; \mathcal{D}_{0, \gamma}^{1, p}(\Omega)\right)}
\end{aligned}
$$

for all $v \in L^{p}\left(\tau, T ; \mathcal{D}_{0, \gamma}^{1, p}(\Omega)\right)$, where we have used the Hölder inequality. Because of the boundedness of $\left\{u_{n}\right\}$ in $L^{p}\left(\tau, T ; \mathcal{D}_{0, \gamma}^{1, p}(\Omega)\right)$, we infer that $\left\{-\Delta_{p, \gamma} u_{n}\right\}$ is bounded in $L^{p^{\prime}}\left(\tau, T ; \mathcal{D}_{-\gamma}^{-1, p^{\prime}}(\Omega)\right)$.

Step 3: Passing limits. From the above estimates, there exists a subsequence $\left\{u_{\mu}\right\} \subset$ $\left\{u_{n}\right\}$ such that

$$
\begin{aligned}
& u_{\mu} \rightarrow u \quad \text { in } L^{p}\left(\tau, T ; \mathcal{D}_{0, \gamma}^{1, p}(\Omega)\right), \\
& \left.f\left(t, u_{\mu}\right) \rightarrow \eta \quad \text { in } L^{q^{\prime}}\left(\tau, T ; L^{q^{\prime}} \Omega\right)\right) \\
& -\Delta_{p, \gamma} u_{\mu} \rightarrow \psi \quad \text { in } L^{p^{\prime}}\left(\tau, T ; \mathcal{D}_{-\gamma}^{-1, p^{\prime}}(\Omega)\right),
\end{aligned}
$$

up to a subsequence.

To prove that $\eta(t)=f(t, u(t))$, we argue similarly to $[22,23]$ to deduce that

$$
\lim _{a \rightarrow 0} \sup _{\mu} \int_{\tau}^{T-a}\left\|u_{\mu}(t+a)-u_{\mu}(t)\right\|_{L^{2}(\Omega)}^{2} d t=0,
$$

for all $T>\tau$. In particular, we obtain from (2.5) that

$$
\lim _{a \rightarrow 0} \sup _{\mu}\left(\int_{\tau}^{T+a}\left\|u_{\mu}(t)\right\|_{L^{2}(\Omega)}^{2} d t+\int_{T-a}^{T}\left\|u_{\mu}(t)\right\|_{L^{2}(\Omega)}^{2} d t\right)=0 .
$$

Then, by Theorem 13.3 and Remark 13.1 in [24], we obtain that $u_{\mu} \rightarrow u$ strongly in $L^{2}\left(\tau, T ; L^{2}(\Omega)\right)$, up to a subsequence. Hence, we can assume that $u_{\mu} \rightarrow u$ a.e. in $Q_{\tau, T}$. Therefore, $f\left(t, u_{\mu}\right) \rightarrow f(t, u)$ a.e. in $Q_{\tau, T}$ since $f$ is continuous. By Lemma 1.3 in [[21], 
Chapter 1], one has

$$
f\left(t, u_{\mu}\right) \rightarrow f(t, u) \quad \text { in } L^{q^{\prime}}\left(\tau, T ; L^{q^{\prime}}(\Omega)\right) .
$$

Thus, we have

$$
\frac{d u}{d t}=\psi-f(t, u)+g(t) \text { in } V^{\prime} .
$$

We now show that $\psi=-\Delta_{p}, \gamma u$. Since $-\Delta_{p}, \gamma$ is monotone, we have

$$
X_{n}=\int_{\tau}^{T}\left\langle-\Delta_{p, \gamma} u_{n}+\Delta_{p, \gamma} v, u_{n}-v\right\rangle d t \geq 0, \quad \text { for all } v \in V .
$$

Note that $\left\{u_{n}(T)\right\}$ is bounded in $L^{2}(\Omega)$, so by arguments as in [[21], pp. 159-160], we have that $u_{n}(T) \rightarrow u(T)$ in $L^{2}(\Omega)$. Because

$$
\begin{aligned}
\int_{\tau}^{T}\left\langle-\Delta_{p, \gamma} u_{n}, u_{n}\right\rangle d t= & -\int_{\tau}^{T} \int_{\Omega}\left(f\left(t, u_{n}\right) u_{n}-g(t) u_{n}\right) d x d t \\
& +\frac{1}{2}\left\|u_{n}(\tau)\right\|_{L^{2}(\Omega)}^{2}-\frac{1}{2}\left\|u_{n}(T)\right\|_{L^{2}(\Omega)}^{2},
\end{aligned}
$$

we obtain

$$
\begin{aligned}
\limsup _{n \rightarrow \infty} X_{n} \leq & -\int_{\tau}^{T}\left(f(t, u) u d t+\frac{1}{2}\|u(\tau)\|_{L^{2}(\Omega)}^{2}-\frac{1}{2}\|u(T)\|_{L^{2}(\Omega)}^{2}\right. \\
& -\int_{\tau}^{T}(\psi, v) d t+\int_{\tau}^{T}\left(\Delta_{p, \gamma} v, u-v\right) d t+\int_{\tau}^{T}(g(t), u) d t
\end{aligned}
$$

where we have used the facts that $u_{n}(\tau) \rightarrow u_{\tau}$ in $L^{2}(\Omega),\|u(T)\|_{L^{2}(\Omega)}^{2} \leq \liminf _{n \rightarrow \infty}\left\|u_{n}(T)\right\|_{L^{2}(\Omega)}^{2}$. On the other hand, by integrating by parts, from (2.14) we have

$$
-\int_{\tau}^{T}(f, u) d t+\frac{1}{2}\|u(\tau)\|_{L^{2}(\Omega)}^{2}-\frac{1}{2}\|u(T)\|_{L^{2}(\Omega)}^{2}+\int_{\tau}^{T}(g(t), u) d t=\int_{\tau}^{T}(\psi, u) d t,
$$

and therefore thanks to (2.15) and (2.16) one gets

$$
\int_{\tau}^{T}\left(\psi+\Delta_{p, \gamma} v, u-v\right) d t \geq 0, \quad \forall v \in V
$$

We now use the hemicontinuity of the operator $\Delta_{p, \gamma}$ to show that $\psi=-\Delta_{p, \gamma} u$. Taking $v=u-\lambda w$, where $\lambda>0$ and $w \in V:=L^{p}\left(\tau, T ; \mathcal{D}_{0, \gamma}^{1, p}(\Omega)\right)$, we obtain

$$
\lambda \int_{\tau}^{T}\left(\psi+\Delta_{p, \gamma}(u-\lambda w), w\right) d t \geq 0
$$


hence

$$
\int_{\tau}^{T}\left(\psi+\Delta_{p, \gamma}(u-\lambda w), w\right) d t \geq 0,
$$

leting $\lambda \rightarrow 0$ in (2.17), we conclude that

$$
\int_{\tau}^{T}\left(\psi+\Delta_{p, \gamma} u, w\right) d t \geq 0, \quad \text { for all } w \in V .
$$

So $\psi=-\Delta_{p, \gamma} u$. Thus,

$$
u^{\prime}=\Delta_{p, \gamma} u-f(t, u)+g(t) \text { in } V^{\prime} .
$$

We now show that $u(\tau)=u_{\tau}$. Choosing some $\varphi \in C^{1}\left([\tau, T] ; \mathcal{D}_{0, \gamma}^{1, p}(\Omega) \cap L^{q}(\Omega)\right)$ with $\phi(T)=0$, observe that $\phi \in V$, by the Lebesgue dominated theorem, one can check that

$$
\begin{aligned}
-\int_{\tau}^{T}\left(u, \varphi^{\prime}\right) d t+\int_{\tau}^{T} \int_{\Omega}|x|^{-p \gamma}|\nabla u|^{p-2} \nabla u \nabla \varphi d x d t & +\int_{\tau}^{T} \int_{\Omega} f(t, u) \varphi d x d t \\
& =(u(\tau), \varphi(\tau))+\int_{\tau}^{T} \int_{\Omega} g \varphi d x d t .
\end{aligned}
$$

Doing the same in the Galerkin approximations yields

$$
\begin{aligned}
-\int_{\tau}^{T}\left(u_{n}, \varphi^{\prime}\right) d t+\int_{\tau}^{T} \int_{\Omega}|x|^{-p \gamma}\left|\nabla u_{n}\right|^{p-2} \nabla u_{n} \nabla \varphi d x d t & +\int_{\tau}^{T} \int_{\Omega} f\left(t, u_{n}\right) \varphi d x d t \\
& =\left(u_{n}(\tau), \varphi(\tau)\right)+\int_{\tau}^{T} \int_{\Omega} g \varphi d x d t .
\end{aligned}
$$

Passing to the limit as $n \rightarrow \infty$, we have

$$
\begin{aligned}
-\int_{\tau}^{T}\left(u, \varphi^{\prime}\right) d t+\int_{\tau}^{T} \int_{\Omega}|x|^{-p \gamma}|\nabla u|^{p-2} \nabla u \nabla \varphi d x d t & +\int_{\tau}^{T} \int_{\Omega} f(t, u) \varphi d x d t \\
& =\left(u_{\tau}, \varphi(\tau)\right)+\int_{\tau}^{T} \int_{\Omega} g \varphi d x d t .
\end{aligned}
$$

Therefore, $u(\tau)=u_{\tau}$ and $u$ is a weak solution of (1.1) on $(\tau, T)$.

Finally, it is easy to check that the solution $u$ satisfies the inequality similar to (2.3), and this implies that the solution $u$ exists globally on the interval $(\tau,+\infty)$.

\section{Existence of global attractors}

\subsection{The autonomous case}

Consider the case where $f$ and $g$ do not depend on the time $t$, and let us recall the definition of multi-valued semiflows. 
Definition 3.1. [5]Let E be a Banach space. The mapping

$$
\mathcal{G}:[0,+\infty) \times E \rightarrow 2^{E}
$$

is called a multi-valued semiflow if the following conditions are satisfied:

(1) $\mathcal{G}(0, w)=w$ for arbitrary $w \in E$;

(2) $\mathcal{G}\left(t_{1}+t_{2}, w\right) \subset \mathcal{G}\left(t_{1}, \mathcal{G}\left(t_{2}, w\right)\right)$ for all $w \in E, t_{1}, t_{2} \in \mathbb{R}^{+}$, where $G(t, B)=\cup_{x \in B}$

$G(t, x), B \subset E$.

It is called a strict multi-valued semiflow if $\mathcal{G}\left(t_{1}+t_{2}, w\right)=\mathcal{G}\left(t_{1}, \mathcal{G}\left(t_{2}, w\right)\right)$, for all $w \in$ $E, t_{1}, t_{2} \in \mathbb{R}^{+}$.

We now consider problem (1.1) with $\tau=0$. By Theorem 2.1, we construct a multivalued mapping as follows

$$
\mathcal{G}\left(t, u_{0}\right)=\left\{u(t) \mid u(\cdot) \text { is a global weak solution of (1.1) such that } u(0)=u_{0}\right\} .
$$

Lemma 3.1. $\mathcal{G}$ is a strict multi-valued semiflow in the sense of Definition 3.1.

Proof. Assume that $\xi \in \mathcal{G}\left(t_{1}+t_{2}, u_{0}\right)$, then $\xi=u\left(t_{1}+t_{2}\right)$, where $u(t)$ is a solution of (1.1). Denoting $v(t)=u\left(t+t_{2}\right)$, we see that $v($.$) is also in the set of solutions of (1.1) with respect$ to initial condition $v(0)=u\left(t_{2}\right)$. Therefore, $\xi=v\left(t_{1}\right) \in \mathcal{G}\left(t_{1}, u\left(t_{2}\right)\right) \subset \mathcal{G}\left(t_{2}, \mathcal{G}\left(t_{2}, u_{0}\right)\right)$. It remains to show that $\mathcal{G}\left(t_{1}, \mathcal{G}\left(t_{2}, u_{0}\right)\right) \subset \mathcal{G}\left(t_{1}+t_{2}, u_{0}\right)$. If $\xi \in \mathcal{G}\left(t_{1}, \mathcal{G}\left(t_{2}, u_{0}\right)\right)$ then $\xi=v$ $\left(t_{1}\right)$, where $v(0) \in \mathcal{G}\left(t_{2}, u_{0}\right)$. One can suppose that $v(0)=u\left(t_{2}\right)$, where $u(0)=u_{0}$. Set

$$
w(\tau)=\left\{\begin{array}{l}
u(\tau), \quad 0 \leq \tau<t_{2} \\
v\left(\tau-t_{2}\right), \tau \geq t_{2}
\end{array}\right.
$$

Since $u$ and $v$ are two solutions of (1.1), we obtain that $w$ is a solution of (1.1) with $w(0)=u(0)=u_{0}$. In addition, since $\xi=v\left(t_{1}\right)=w\left(t_{1}+t_{2}\right)$, we have $\xi \in \mathcal{G}\left(t_{1}+t_{2}, u_{0}\right)$.

Definition 3.2. [5] A set $\mathcal{A}$ is said to be a global attractor of the multi-valued semiflow $\mathcal{G}$ if the following conditions hold:

- $\mathcal{A}$ is an attracting, i.e., $\operatorname{dist}(\mathcal{G}(t, B), \mathcal{A}) \rightarrow 0$ as $t \rightarrow \infty$ for all bounded subsets $B$ $\subset E$,

- $\mathcal{A}$ is negatively semi-invariant: $\mathcal{A} \subset \mathcal{G}(t, \mathcal{A})$ for arbitrary $t \geq 0$,

- If $\mathscr{B}$ is an attracting of $\mathcal{G}$ then $\mathcal{A} \subset \overline{\mathcal{B}}$,

$$
\text { where } \operatorname{dist}(\mathcal{C}, \mathcal{A})=\sup _{c \in \mathcal{C}} \inf _{a \in \mathcal{A}}\|c-a\| \text { is the Hausdorff semi-distance. }
$$

The following theorem gives the sufficient conditions for the existence of a global attractor for the multi-valued semiflow $\mathcal{G}$.

Theorem 3.2. [5,7]Suppose that the strict multi-valued semiflow $\mathcal{G}$ has the following properties:

(1) $\mathcal{G}$ is pointwise dissipative, i.e., there exists $K>0$ such that for $u_{0} \in E, u(t) \in \mathcal{G}\left(t, u_{0}\right)$ one has $\|u(t)\|_{E} \leq K$ if $t \geq t_{0}\left(\left\|u_{0}\right\|_{E}\right)$;

(2) $\mathcal{G}(t,$.$) is a closed map for any t \geq 0$, i.e., if $\xi_{n} \rightarrow \xi, \eta_{n} \rightarrow \eta, \xi_{n} \in \mathcal{G}\left(t, \eta_{n}\right)$ then $\xi \in \mathcal{G}(t, \eta)$; 
(3) $\mathcal{G}$ is asymptotically upper semicompact, i.e., if $B$ is a bounded set in $E$ such that for some $T(B), \gamma_{T(B)}^{+}(B):=\bigcup_{t \geq T(B)} \mathcal{G}(t, B)$ is bounded, any sequence $\xi_{n} \in \mathcal{G}\left(t_{n}, B\right)$ with $t_{n} \rightarrow \infty$ is precompact in $E$.

Then $\mathcal{G}$ has a compact global attractor $\mathcal{A}$ in E. Moreover, $\mathcal{A}$ is invariant, i.e., $\mathcal{G}(t, \mathcal{A})=\mathcal{A}$ for any $t \geq 0$.

Lemma 3.3. $\mathcal{G}\left(t^{*},.\right)$ is a compact mapping for each $t^{*} \in(0, T]$.

Proof. This lemma is a direct consequence of Lemma 3.8 in Section 3.2 below.

We now can prove the existence of a global attractor.

Theorem 3.4. Under conditions (H1)-(H3), where $f$ andg are assumed to be independent of time $t$, the strict multi-valued semiflow $\mathcal{G}$ generated by problem (1.1) has an invariant compact global attractor in $L^{2}(\Omega)$.

Proof. We will check hypotheses (1)-(3) of Theorem 3.2. First, assume $u(t) \in \mathcal{G}\left(t, u_{0}\right)$, we have

$$
\begin{aligned}
\frac{1}{2} \frac{d}{d t}\|u(t)\|_{L^{2}(\Omega)}^{2}+\int_{\Omega}|x|^{-p \gamma}|\nabla u|^{p}+C_{2}\|u\|_{L^{q}(\Omega)}^{q} & \leq k_{2}|\Omega|+\int_{\Omega} u g d x \\
& \leq k_{2}|\Omega|+\varepsilon\|u\|_{L^{2}(\Omega)}^{2}+C_{\varepsilon}\|g\|_{L^{2}(\Omega)}^{2} .
\end{aligned}
$$

Noting that

$$
C_{2}\|u\|_{L^{q}(\Omega)}^{q} \geq \lambda\|u\|_{L^{2}(\Omega)}^{2}-C, \quad C=C(q,|\Omega|)>0,
$$

we have

$$
\frac{1}{2} \frac{d}{d t}\|u\|_{L^{2}(\Omega)}^{2}+\lambda\|u\|_{L^{2}(\Omega)}^{2} \leq C\left(q,|\Omega|,\|g\|_{L^{2}(\Omega)}\right) .
$$

Therefore

$$
\|u(t)\|_{L^{2}(\Omega)}^{2} \leq\|u(0)\|_{L^{2}(\Omega)}^{2} e^{-2 \lambda t}+C\left(q,|\Omega|,\|g\|_{L^{2}(\Omega)}\right),
$$

Hence one can deduce that $\mathcal{G}$ is pointwise dissipative.

We now check hypothesis (2) of Theorem 3.2. Assume that $\xi_{n} \in \mathcal{G}\left(t, \eta_{n}\right), \xi_{n} \rightarrow \xi, \eta_{n} \rightarrow \eta$ in $L^{2}(\Omega)$. Then there exists a sequence $\left\{u_{n}\right\}$ such that

$$
u_{n}(t)=\xi_{n}, \quad u_{n}(0)=\eta_{n} .
$$

Using the same arguments as in the proof of Theorem 2.1, we have

- $u_{n} \rightarrow u$ in $L^{2}\left(Q_{0, T}\right)$

- $u_{n}(t)-u(t)$ in $L^{2}(\Omega)$ for arbitrary $t \in[0, T]$ (and then $u(0)=\eta$ ),

- $f\left(u_{n}\right) \rightarrow f(u)$ in $L^{q^{\prime}}\left(Q_{0, T}\right)$,

- $\frac{d u_{n}}{d t} \rightarrow \frac{d u}{d t}$ in $V$

- $-\Delta_{p, \gamma} u_{n}-\Delta_{p, \gamma} u$ in $L^{p^{\prime}}\left(0, T ; \mathcal{D}_{-\gamma}^{-1, p^{\prime}}(\Omega)\right)$,

up to a subsequence. Hence, passing to the limit in the equality 


$$
\int_{0}^{T}\left\langle u_{n}^{\prime}, v\right\rangle+\int_{0}^{T} d t \int_{\Omega}|x|^{-p \gamma}\left|\nabla u_{n}\right|^{p-2} \nabla u_{n} \nabla v+\int_{0}^{T} d t \int_{\Omega} f\left(u_{n}\right) v=\int_{0}^{T} d t \int_{\Omega} g v
$$

we conclude that $u(t)$ is a weak solution of (1.1) with the initial condition $u(0)=\eta$. Thus, $\xi \in \mathcal{G}(t, \eta)$.

For hypothesis (3), one observes that for $n$ large enough,

$$
\mathcal{G}\left(t_{n}, B\right)=\mathcal{G}\left(t^{*}+t_{n}-t^{*}, B\right) \subset \mathcal{G}\left(t^{*}, \mathcal{G}\left(t_{n}-t^{*} B\right)\right) \subset \mathcal{G}\left(t^{*}, B^{*}\right),
$$

where $t^{*}>0$ and $B^{*}$ is a bounded set in $L^{2}(\Omega)$. Using Lemma 3.3, we conclude that, if $\xi_{n} \in \mathcal{G}\left(t_{n}, B\right)$, then $\left\{\xi_{n}\right\}$ is precompact in $L^{2}(\Omega)$.

\subsection{The non-autonomous case}

Let us recall some definitions and related results. The pair of functions $(f(s, \cdot), g(\cdot, s))=\sigma$ $(s)$ is called a symbol of (1.1). We consider (1.1) with a family of symbols including the shifted forms $\sigma(s+h)=(f(s+h, \cdot), g(\cdot, s+h))$ and the limits of some sequence $\{\sigma(s+$ $\left.\left.h_{n}\right)\right\}_{n \in N}$ in an appropriate topological space $\Sigma$. The family of such symbols is said to be the hull of $\sigma$ in $\Sigma$ and is denoted by $\mathcal{H}(\sigma)$, i.e.,

$$
\mathcal{H}(\sigma)=c l_{\sum}\{\sigma(\cdot+h) \mid h \in \mathbb{R}\}
$$

If the hull $\mathcal{H}(\sigma)$ is a compact set in $\Sigma$, we say that $\sigma$ is translation compact in $\Sigma$.

Denote $\mathbb{R}_{d}=\left\{(t, \tau) \in \mathbb{R}^{2} \mid \tau \leq t\right\}$. Let $X$ be a complete metric space, $\mathcal{P}(X)$ and $\mathcal{B}(X)$ be the set of all nonempty subsets and the set of all nonempty bounded subsets of the space $X$, respectively and let $\Sigma$ be a subspace of $\Sigma$.

Denote

$$
\begin{gathered}
Z=\left\{\varphi \in C(\mathbb{R} ; \mathbb{R}):|\varphi(u)| \leq C_{\varphi}\left(1+|u|^{q-1}\right), \text { for some } C_{\varphi}>0\right\}, \\
\|\varphi\|_{Z}=\sup _{u \in \mathbb{R}} \frac{|\varphi(u)|}{1+|u|^{q-1}} .
\end{gathered}
$$

Then $Z$ is a Banach space. We say that $f_{n} \rightarrow f$ in the space $C(\mathbb{R} ; Z)$ if

$$
\lim _{n \rightarrow \infty} \sup _{s \in[t, t+r]}\left\|f_{n}(s, \cdot)-f(s, \cdot)\right\|_{Z}=0
$$

for all $t \in \mathbb{R}, r>0$.

Let $f_{0} \in C(\mathbb{R} ; Z), g_{0} \in L_{\text {loc }}^{2, w}\left(\mathbb{R} ; L^{2}(\Omega)\right)$, and

$$
\begin{aligned}
& \mathcal{H}\left(f_{0}\right)=\operatorname{cl}_{C(\mathbb{R} ; Z)}\left\{f_{0}(\cdot+h) \mid h \in \mathbb{R}\right\}, \\
& \mathcal{H}\left(g_{0}\right)=\operatorname{cl}_{L_{\text {loc }}^{2, w}\left(\mathbb{R} ; L^{2}(\Omega)\right)}\left\{g_{0}(\cdot+h) \mid h \in \mathbb{R}\right\},
\end{aligned}
$$

where the topology in $L_{\text {loc }}^{2, w}\left(\mathbb{R} ; L^{2}(\Omega)\right)$ is equipped by the local weak convergence, i.e., $g_{n} \rightarrow g$ in $L_{\text {loc }}^{2, w}\left(\mathbb{R} ; L^{2}(\Omega)\right)$ if

$$
\lim _{n \rightarrow \infty} \int_{t}^{t+r} \int_{\Omega}\left(g_{n}(s, x)-g(s, x)\right) \phi(x, s) d s d x=0
$$

for all $t \in \mathbb{R}, r>0$ and $\varphi \in L^{2}\left(Q_{t, t+r}\right)$. We define $\sum=\mathcal{H}\left(f_{0}\right) \times \mathcal{H}\left(g_{0}\right)$. 
In order to deal with a uniform attractor with respect to the family of symbols, one usually requires the translation compact property. Let us recall some discussions on this requirement. It is known that hypothesis (H2) ensures that $g$ is translation compact in $L_{\text {loc }}^{2, w}\left(\mathbb{R} ; L^{2}(\Omega)\right.$ ) (see [4] for details). In addition, the following statement gives a sufficient condition for the translation compact property in $C(\mathbb{R} ; Z)$.

Proposition 3.5. [4] The function $f \in C(\mathbb{R} ; Z)$ is translation compact if and only if for all $R>0$ one has

(1) $|f(t, v)| \leq C(R)$ for all $t \in \mathbb{R}, v \in[-R, R]$,

(2) $\left|f\left(t_{1}, v_{1}\right)-f\left(t_{2}, v_{2}\right)\right| \leq \alpha\left(\left|t_{1}-t_{2}\right|+\left|v_{1}-v_{2}\right|, R\right), \forall t_{1}, t_{2} \in \mathbb{R}, v_{1}, v_{2} \in[-R, R]$, here $C(R)$

$>0$ and $\alpha(.,$.$) is a function such that \alpha(s, R) \rightarrow 0$ as $s \rightarrow 0^{+}$.

From now on, we suppose that $f$ is translation compact. Together with the fact that $g$ is translation compact in $L_{\text {loc }}^{2, w}\left(\mathbb{R} ; L^{2}(\Omega)\right)$, one sees that $\Sigma$ is a compact set in $L_{\text {loc }}^{2, w}\left(\mathbb{R} ; L^{2}(\Omega)\right)$. Then it follows from [4] that $T(h): \Sigma \rightarrow \Sigma$ is continuous and $T(h) \Sigma \subset$ $\Sigma$ for all $h \in \mathbb{R}$.

Definition 3.3. [6] The map $U: \mathbb{R}_{d} \times X \rightarrow \mathcal{P}(X)$ is called an multi-valued semiprocess (MSP) if

(1) $U(\tau, \tau,)=.I d$ (the identity map),

(2) $U(t, \tau, x) \subset U(t, s, U(s, \tau, x))$, for all $x \in X, t, s, \tau \in \mathbb{R}, \tau \leq s \leq t$.

It is called a strict multi-valued semiprocess if $U(t, \tau, x)=U(t, s, U(s, \tau, x))$.

We denote by $\mathcal{D}_{\tau, \sigma}\left(u_{\tau}\right)$ the set of all global weak solutions (defined for all $t \geq \tau$ ) of the problem (1.1) with data $\left(f_{\sigma}, g_{\sigma}\right)$ instead of $(f, g)$ such that $u(\tau)=u_{\tau}$. For each $\sigma=(f$, $g) \in \Sigma$, we consider the family of MSP $\left\{U_{\sigma}: \sigma \in \Sigma\right\}$ defined by

$$
U_{\sigma}\left(t, \tau, u_{\tau}\right)=\left\{u=u(t) \mid u(\cdot) \in \mathcal{D}_{\tau, \sigma}\left(u_{\tau}\right)\right\}
$$

Lemma 3.6. $U_{\sigma}\left(t, \tau, u_{\tau}\right)$ is a multi-valued semiprocess. Moreover,

$$
U_{\sigma}\left(t+s, \tau+s, u_{\tau}\right)=U_{T(s) \sigma}\left(t, \tau, u_{\tau}\right) \text { for all } u_{\tau} \in L^{2}(\Omega),(t, \tau) \in \mathbb{R}_{d}, s \in \mathbb{R} .
$$

Proof. Given $\left.z \in U_{\sigma}\left(t, \tau, u_{\tau}\right)\right)$ we have to prove that $z \in U_{\sigma}\left(t, s, U_{\sigma}\left(s, \tau, u_{\tau}\right)\right)$. Take $y(.) \in \mathcal{D}_{\tau, \sigma}\left(u_{\tau}\right)$ such that $y(\tau)=u_{\tau}$ and $y(t)=z$. Clearly, $y(s) \in U_{\sigma}\left(s, \tau, u_{\tau}\right)$. Then if we define $z(t)=y(t)$ for $t \geq s$ we have that $z(s)=y(s)$ and obviously $z(.) \in \mathcal{D}_{s, \sigma}(\gamma(s))$. Consequently, $z(t) \in U_{\sigma}\left(t, s, U_{\sigma}\left(s, \tau, u_{\tau}\right)\right)$.

Let $z \in U_{\sigma}\left(t+s, \tau+s, u_{\tau}\right)$. Then there exists $u(\cdot) \in \mathcal{D}_{\tau+s, \sigma}\left(u_{\tau}\right)$ such that $z=u(t+s)$ and $v(\cdot)=u(\cdot+s) \in \mathcal{D}_{\tau, T(s) \sigma}\left(u_{\tau}\right)$, so that $z=v(t) \in u_{\tau, T}(s) \sigma\left(u_{\tau}\right)$.

Conversely, if $z \in U_{\tau, T(s) \sigma}\left(u_{\tau}\right)$, then there is $z \in \mathcal{D}_{\tau, T(s) \sigma}\left(u_{\tau}\right)$ such that $z=u(t)$ and $v(\cdot)=u(-s+\cdot) \in \mathcal{D}_{\tau+s, \sigma}\left(u_{\tau}\right)$ so that $z=v(t+s) \in U_{\sigma}\left(t+s, \tau+s, u_{\tau}\right)$.

Denote by

$$
U_{\Sigma}(t, \tau, x)=\bigcup_{\sigma \in \Sigma} U_{\sigma}(t, \tau, x)
$$


Definition 3.4. [6]A set $\mathcal{A}$ is called a uniform global attractor for the family of multi-valued semiprocesses $U_{\Sigma}$ if:

(1) it is negatively semiinvariant, i.e., $\mathcal{A} \subset U_{\Sigma}(t, \tau, \mathcal{A})$ for all $t \geq \tau$;

(2) it is uniformly attracting, i.e., $\operatorname{dist}\left(U_{\Sigma}(t, \tau, B), \mathcal{A}\right) \rightarrow 0$, as $t \rightarrow \infty$, for all

$B \in \mathcal{B}(X)$ and $\tau \in \mathbb{R}$;

(3) for any closed uniformly attracting set $Y$, we have $\mathcal{A} \subset Y$ (minimality).

Theorem 3.7. [[6], Theorem 2] Suppose that the family of multi-valued semiprocesses $U_{\Sigma}$ satisfies the following conditions:

(1) On $\Sigma$ is defined the continuous shift operator $T(s) \sigma(t)=\sigma(t+s)$, $s \in \mathbb{R}$ such that $T(h) \Sigma \subset \Sigma$, and for any $(t, \tau) \in \mathbb{R}_{d}, \sigma \in \Sigma, s \in \mathbb{R}, x \in X$, we have

$$
U_{\sigma}(t+s, \tau+s, x)=U_{T(s) \sigma}(t, \tau, x)
$$

(2) $U_{\sigma}$ is uniformly asymtopically upper semicompact;

(3) $U_{\sigma}$ is pointwise dissipative;

(4) The map $(x, \sigma) \mapsto U_{\sigma}(t, 0, x)$ has closed values and is w-upper semicontinuous.

Then the family of multi-valued semiprocesses $U_{\Sigma}$ has a uniform global compact attractor $\mathcal{A}$.

The following is the key point of this subsection.

Lemma 3.8. Let conditions (H1)-(H3) hold and let $\left\{u_{n}\right\}_{n \in \mathbb{N}}$ is a sequence of weak solutions of (1.1) with respect to the sequence of symbols $\left\{\sigma_{n}\right\} \subset \Sigma$ such that

$$
\begin{aligned}
& \text { (1) } u_{n}(\tau) \rightarrow u_{\tau} \text { in } L^{2}(\Omega), \\
& \text { (2) } \sigma_{n} \rightarrow \sigma \text { in } \sum .
\end{aligned}
$$

Then there exists a solution $u$ of (1.1) with respect to the symbol $\sigma$ such that $u(\tau)=u_{T}$ and $u_{n}\left(t^{*}\right) \rightarrow u\left(t^{*}\right)$ in $L^{2}(\Omega)$ for any $t^{*}>\tau$, up to a subsequence.

Proof. Let $\sigma_{n}=\left(f_{n}, g_{n}\right)$. Since $f$ satisfies $(H 1)$ for all $t \in \mathbb{R}$ and $f_{n} \in \mathcal{H}(f)$, one sees that $f_{n}$ also satisfies $(H 1)$. On the other hand, noting that $\left\{u_{n}(\tau)\right\}$ is bounded in $L^{2}(\Omega)$ and $\left\|g_{n}\right\|_{L_{b}^{2}} \leq\|g\|_{L_{b}^{2}}$. Thus, repeating the arguments in the proof of Theorem 2.1, we obtain that

$$
\begin{aligned}
& \left\{u_{n}\right\} \text { is bounded in } V=L^{p}\left(\tau, T ; \mathcal{D}_{0, \gamma}^{1, p}(\Omega)\right) \cap L^{q}\left(\tau, T ; L^{q}(\Omega)\right), \\
& \left\{u_{n}^{\prime}\right\} \text { is bounded in } V^{\prime}=L^{p^{\prime}}\left(\tau, T ; \mathcal{D}_{-\gamma}^{-1, p^{\prime}}(\Omega)\right)+L^{q^{\prime}}\left(\tau, T ; L^{q^{\prime}}(\Omega)\right), \\
& \left\{u_{n}\right\} \text { is bounded in } C\left([\tau, T] ; L^{2}(\Omega)\right), \\
& \left\{f_{n}\left(t, u_{n}\right)\right\} \text { is bounded in } L^{q^{\prime}}\left(Q_{\tau, T}\right), \\
& \left\{-\Delta_{p, \gamma} u_{n}\right\} \text { is bounded in } L^{p^{\prime}}\left(\tau, T ; \mathcal{D}_{-\gamma}^{-1, p^{\prime}}(\Omega)\right) .
\end{aligned}
$$


In particular, we have

$$
u_{n}(t) \rightarrow u(t) \text { in } L^{2}(\Omega) \text { for all } t \in[\tau, T],
$$

up to a subsequence. Let $\sigma_{n} \rightarrow \sigma=(\bar{f}, \bar{g})$ in $\Sigma$, to show that $u$ is a solution of (1.1) with respect to the symbol $\sigma$ such that $u(\tau)=u_{T}$, we need to pass to the limits in the following relation

$$
\int_{\tau}^{T} \int_{\Omega}\left(u_{n}^{\prime} v+|x|^{-p \gamma}\left|\nabla u_{n}\right|^{p-2} \nabla u_{n} \nabla v+f_{n}\left(t, u_{n}\right) v\right) d x d t=\int_{\tau}^{T} \int_{\Omega} g_{n} v d x d t
$$

for all $v \in V$. Since $g_{n} \rightarrow \bar{g}$ in $L^{2}\left(\tau, T ; L^{2}(\Omega)\right)$, it remains to prove that $f_{n}\left(t, u_{n}\right) \rightarrow \bar{f}(t, u)$ in $L^{q^{\prime}}\left(Q_{\tau, T}\right)$. We first show that $f_{n}\left(t, u_{n}\right) \rightarrow \bar{f}\left(t, u_{n}\right)$ in $L^{q^{\prime}}\left(Q_{\tau, T}\right)$. Indeed,

$$
\begin{aligned}
& \int_{\tau}^{T} \int_{\Omega}\left|f_{n}\left(t, u_{n}\right)-\bar{f}\left(t, u_{n}\right)\right|^{q^{\prime}} d x d t \\
= & \int_{\tau}^{T} \int_{\Omega} \frac{\left|f_{n}\left(t, u_{n}\right)-\bar{f}\left(t, u_{n}\right)\right|^{q^{\prime}}}{\left(1+\left|u_{n}\right|^{q-1}\right)^{q^{\prime}}}\left(1+\left|u_{n}\right|^{q-1}\right)^{q^{\prime}} d x d t \\
\leq & \left(\sup _{[\tau, T]} \mid f_{n}-\bar{f} \|_{Z}\right)^{q^{\prime}} \int_{\tau}^{T} \int_{\Omega}\left(1+\left|u_{n}\right|^{q}\right) d x d t \rightarrow 0
\end{aligned}
$$

because $f_{n} \rightarrow \bar{f}$ in $Z$ and $\left\{u_{n}\right\}$ is bounded in $L^{q}\left(Q_{\tau, T}\right)$. On the other hand, since $\left\{\bar{f}\left(t, u_{n}\right)\right\}$ is bounded in $L^{q^{\prime}}\left(Q_{\tau, T}\right)$, by using Lemma 1.3 in [[21], Chapter 1$]$ and the continuity of $\bar{f}$ as in the proof of Theorem 2.1, we can conclude that $\bar{f}\left(t, u_{n}\right) \rightarrow \bar{f}(t, u)$ weakly in $L^{q^{\prime}}\left(Q_{\tau, T}\right)$. Hence, we have

$$
f_{n}\left(t, u_{n}\right)-\bar{f}(t, u)=\left(f_{n}\left(t, u_{n}\right)-\bar{f}\left(t, u_{n}\right)\right)+\left(\bar{f}\left(t, u_{n}\right)-\bar{f}(t, u)\right) \rightarrow 0 \text { weakly in } L^{q^{\prime}}\left(Q_{\tau, T}\right) .
$$

We now have to show that $u_{n}\left(t^{*}\right) \rightarrow u\left(t^{*}\right)$ in $L^{2}(\Omega)$ for any $t^{*}>\tau$. Taking into account of (3.3), we have to check that $\left\|u_{n}\left(t^{*}\right)\right\|_{L^{2}(\Omega)} \rightarrow\left\|u\left(t^{*}\right)\right\|_{L^{2}(\Omega)}$.

Putting

$$
\begin{gathered}
J_{n}(t)=\left\|u_{n}(t)\right\|_{L^{2}(\Omega)}^{2}-2 \int_{\tau}^{t}\left(g_{n}(s), u_{n}(s)\right) d s-\left(2 k_{2}|\Omega|+2 \lambda\right)(t-\tau), \\
J(t)=\|u(t)\|_{L^{2}(\Omega)}^{2}-2 \int_{\tau}^{t}(g(s), u(s)) d s-\left(2 k_{2}|\Omega|+2 \lambda\right)(t-\tau) .
\end{gathered}
$$

It is easy to check that the functions $J_{n}(t), J(t)$ are continuous and non-increasing on $[\tau, T]$. We first show that

$$
J_{n}(t) \rightarrow J(t) \text { for a.e. } t \in[\tau, T] .
$$


Indeed,

$$
\begin{aligned}
\left|J_{n}(t)-J(t)\right| \leq & \left|\left\|u_{n}(t)\right\|_{L^{2}(\Omega)}^{2}-\|u(\gamma)\|_{L^{2}(\Omega)}^{2}\right| \\
& +2\left|\int_{\tau}^{t}\left[\left(g_{n}(s), u_{n}(s)\right)-(g(s), u(s))\right] d s\right| \\
\leq & \left\|u_{n}(t)-u(t)\right\|_{L^{2}(\Omega)}\left(\left\|u_{n}(t)\right\|_{L^{2}(\Omega)}+\|u(t)\|_{L^{2}(\Omega)}\right) \\
& +2 \mid \int_{\tau}^{t}\left[\left(g_{n}(s), u_{n}(s)-u(s)\right) d s \mid\right. \\
& +2 \mid \int_{\tau}^{t}\left[\left(g_{n}(s)-g(s), u(s)\right) d s \mid\right.
\end{aligned}
$$

and

$$
\mid \int_{\tau}^{t}\left[\left(g_{n}(s), u_{n}(s)-u(s)\right) d s \mid \leq\left\|g_{n}\right\|_{L^{2}(Q \tau, t)}\left\|u_{n}(t)-u(t)\right\|_{L^{2}(\Omega)} \rightarrow 0\right.
$$

as $n \rightarrow \infty$ since $u_{n} \rightarrow u$ strongly in $L^{2}\left(Q_{\tau, t}\right)$ and $\left\{g_{n}\right\}$ is bounded in $L^{2}\left(Q_{\tau, t}\right)$. In addition,

$$
\int_{\tau}^{t}\left(g_{n}(s)-g(s), u(s)\right) d s \rightarrow 0
$$

as $n \rightarrow \infty$ since $g_{n} \rightarrow g$ in $L^{2}\left(Q_{\tau, t}\right)$. Then (3.4) is proved due to the fact that $u_{n}(t) \rightarrow u$ $(t)$ in $L^{2}(\Omega)$ for a.e. $t \in[\tau$, T].

We choose an increasing sequence $\left\{t_{m}\right\} \subset[\tau, T], t_{m} \rightarrow t^{*}$ such that $J_{n}\left(t_{m}\right) \rightarrow J\left(t_{m}\right)$ as $n$ $\rightarrow \infty$. Then, by the continuity,

$$
J_{n}\left(t_{m}\right) \rightarrow J_{n}\left(t^{*}\right), \quad \text { as } m \rightarrow \infty .
$$

So

$$
J_{n}\left(t^{*}\right)-J\left(t^{*}\right) \leq J_{n}\left(t_{m}\right)-J\left(t^{*}\right)=J_{n}\left(t_{m}\right)-J\left(t_{m}\right)+J\left(t_{m}\right)-J\left(t^{*}\right)<\varepsilon
$$

for $n \geq n_{0}(\varepsilon)$ and any $\varepsilon>0$. Hence, lim sup $J_{n}\left(t^{*}\right) \leq J\left(t^{*}\right)$ and then lim sup $\left\|u_{n}\left(t^{*}\right)\right\| \leq$ $\left\|u\left(t^{*}\right)\right\|$. From the weak convergence $u_{n}\left(t^{*}\right) \rightarrow u\left(t^{*}\right)$ we have then $\left\|u_{n}\left(t^{*}\right)\right\| \rightarrow\left\|u\left(t^{*}\right)\right\|$, so $u_{n}\left(t^{*}\right) \rightarrow u\left(t^{*}\right)$ strongly in $L^{2}(\Omega)$ as $n \rightarrow \infty$. This completes the proof.

Theorem 3.9. Let conditions (H1)-(H3) hold. Then the family of multi-valued semipro-cesses $\left\{U_{\sigma}(t, \tau)\right\}$ has a uniform global compact attractor $\mathcal{A}$.

Proof. We know that each symbol $\sigma_{n}=\left(f_{n}, g_{n}\right) \in \Sigma$ satisfies the same conditions as in $(H 1)$ - $(H 2)$. Furthermore, since $g_{n} \in \mathcal{H}(g)$, we have $\left\|g_{n}\right\|_{L_{b}^{2}} \leq\|g\|_{L_{b}^{2}}$. Hence if $u_{n}$ is a weak solution of (1.1) with respect to the symbol $\sigma_{n}$, one has

$$
\left\|u_{n}(t)\right\|_{L^{2}(\Omega)}^{2} \leq\left\|u_{n}(\tau)\right\|_{L^{2}(\Omega)}^{2} e^{-\lambda(t-\tau)}+\frac{1}{\lambda\left(1-e^{-\lambda}\right)}\|g\|_{L_{b}^{2}}^{2}+\frac{2 k_{2}|\Omega|}{\lambda}+2 .
$$


The last inequality ensures the existence of a positive number $R_{0}$ such that if $u_{n}(\tau) \in$ $B_{R}$, the ball in $L^{2}(\Omega)$ centered at 0 with radius $R$, then there exists $T_{0}=T_{0}(\tau, R)$ such that

$$
u_{n}(t) \in B_{R_{0}} \quad \text { for all } t \geq T_{0},
$$

that is, $U_{\Sigma}\left(t, \tau, B_{R}\right) \subset B_{R_{0}}$, for all $t \geq T_{0}(\tau, R)$. Thus, $\left\{U_{\sigma}(t, \tau)\right\}$ fulfills condition (3) in Theorem 3.7.

We now define the set $K=\overline{U_{\Sigma}\left(1,0, B_{R_{0}}\right)}$. Lemma 3.8 implies that $K$ is compact. Moreover, since $B_{R_{0}}$ is an absorbing set, we have

$$
\begin{aligned}
U_{\sigma_{n}}\left(t, \tau, B_{R}\right) & =U_{\sigma_{n}}\left(t, t-1, U_{\sigma_{n}}\left(t-1, \tau, B_{R}\right)\right. \\
& =U_{T(t-1) \sigma_{n}}\left(1,0, U_{T(\tau) \sigma_{n}}\left(t-1-\tau, 0, B_{R}\right)\right) \\
& \subset U_{\Sigma}\left(1,0, B_{R_{0}}\right) \subset K
\end{aligned}
$$

for all $\sigma_{n} \in \sum, B_{R} \in \mathcal{B}\left(L^{2}(\Omega)\right)$, and $t \geq T_{0}\left(\tau, B_{R}\right)$. It follows that any sequence $\left\{\xi_{n}\right\}$ such that $\left\{\xi_{n}\right\} \in U_{\sigma_{n}}\left(t_{n}, \tau, B_{R_{0}}\right), \sigma_{n} \in \sum, t_{n} \rightarrow+\infty, B_{R} \in \mathcal{B}\left(L^{2}(\Omega)\right)$, is precompact in $L^{2}$ $(\Omega)$. It is a consequence of Lemma 3.8 that the map $U_{\sigma}$ has compact values for any $\sigma$ $\in \Sigma$.

Finally, let us prove that the map $(\sigma, x) \mapsto U_{\sigma}(t, \tau, x)$ is upper semicontinuous for each fixed $t \geq \tau$. Suppose that it is not true, that is, there exist $\bar{u} \in L^{2}(\Omega), t \geq \tau, \bar{\sigma} \in \sum, \varepsilon>0, \delta_{n} \rightarrow 0, u_{n} \in B_{\delta_{n}}(\bar{u}), \sigma_{n} \rightarrow \bar{\sigma}, \quad$ and $\quad \xi_{n} \in U_{\sigma_{n}}\left(t, \tau, u_{n}\right)$ such that $\left\{\xi_{n}\right\} \notin B_{\varepsilon}\left(U_{\bar{\sigma}}(t, \tau, \bar{u})\right.$. But Lemma 3.8 implies (up to a subsequence) that $\xi_{n} \rightarrow \xi \in U_{\bar{\sigma}}(t, \tau, \bar{u})$, which is a contracdition. Thus, the existence of the uniform global compact attractor follows then from Theorem 3.7.

\section{Acknowledgements}

This work was supported by Vietnam's National Foundation for Science and Technology Development (NAFOSTED), Project 101.01-2010.05.

The authors would like to thank the reviewers for valuable comments and suggestions.

\section{Author details}

${ }^{1}$ Department of Applied Mathematics and Informatics, Hanoi University of Science and Technology, 1 Dai Co Viet, Hai Ba Trung, Hanoi, Vietnam ²Department of Mathematics, Hanoi National University of Education, 136 Xuan Thuy, Cau Giay, Hanoi, Vietnam

\section{Authors' contributions}

All authors read and approved the final manuscript.

\section{Competing interests}

The authors declare that they have no competing interests.

Received: 21 February 2011 Accepted: 28 March 2012 Published: 28 March 2012

\section{References}

1. Chepyzhov, W, Vishik, MI: Attractors for Equations of Mathematical Physics. In Am Math Soc Collog Publ Am Math Soc, vol. 49,Providence, RI (2002)

2. Temam, R: Infinite Dimensional Dynamical Systems in Mechanics and Physics. Springer-Verlag, New York, 2 (1997)

3. Ball, JM: Continuity properties and global attractor of generalized semiflows and the Navier-Stokes equations. J Nonlinear Sci. 7, 475-502 (1997)

4. Chepyzhov, W, Vishik, Ml: Evolution equations and their trajectory attractor. J Math Pure Appl. 76, 913-964 (1997)

5. Melnik, VS, Valero, J: On attractors of multi-valued semiflows and differential inclusions. Set Valued Anal. 6, 83-111 (1998)

6. Melnik, VS, Valero, J: On global attractors of multi-valued semiprocesses and nonautonomous evalution inclusions. Set Valued Anal. 8, 375-403 (2000)

7. Melnik, VS, Valero, J: Addendum to On attractors of multi-valued semiflows and differential inclusions. Set Valued Anal. $16,507-509(2008)$ 
8. Kapustyan, AV: Global attractors of a nonautonomous reaction-diffusion equation. Diff Equ 38(10):1467-1471 (2002). [Translation from Differensial?'?nye Uravneniya 38(10), 1378-1381 (2002)]

9. Kapustyan, AV, Shkundin, DV: Global attractor of one nonlinear parabolic equation. Ukrain Math Zh. 55, $446-455$ (2003)

10. Valero, J, Kapustyan, A: On the connectedness and asymptotic behaviour of solutions of reaction-diffusion systems. J Math Anal Appl. 323, 614-633 (2006)

11. Kapustyan, AV, Menik, VS, Valero, J: Attractors of multivalued dynamical processes generated by phase-field equations. Int J Bifur Chaos. 13, 1969-1984 (2003)

12. Ball, JM: Global attractor for damped semilinear wave equations. Discret Cont Dyn Syst. 10, $31-52$ (2004)

13. Sell, G: Global attractor for the three-dimensional Navier-Stokes equation. J Dyn Diff Equ. 8, 1-33 (1996)

14. Caffarelli, L, Kohn, R, Nirenberg, L: First order interpolation inequalities with weights. Compositio Math. 53, 259-275 (1984)

15. Abdellaoui, B, Colorado, E, Peral, I: Existence and nonexistence results for a class of linear and semi-linear parabolic equations related to some Caffarelli-Kohn-Nirenberg inequalities. J Eur Math Soc. 6, 119-148 (2004)

16. Abdellaoui, B, Peral, I: Harnack inequality for degenerate parabolic equations related to Caffarelli-Kohn-Nirenberg inequalities. Nonlinear Anal. 57, 971-1003 (2004)

17. Abdellaoui, B, Peral, I: Competition reaction-absorption in some elliptic and parabolic problems related to the CaffarelliKohn-Nirenberg inequalities. J Math Anal Appl. 314, 590-617 (2006)

18. Abdellaoui, B, Peral, I: The effect of Harnack inequality on the existence and nonexistence results for quasi-linear parabolic equations related to Caffarelli-Kohn-Nirenberg inequalities. NoDEA Nonlinear Diff Equ Appl. 14, 335-360 (2007)

19. Dall'aglio, A, Giachetti, D, Peral, I: Results on parabolic equations related to some Caffarelli-Kohn-Nirenberg inequalities SIAM Math Anal. 36, 691-716 (2004)

20. Morillas, F, Valero, J: Attractors for reaction-diffusion equations in $\mathbb{\Xi}^{N}$ with continuous nonlinearity. Asymptot Anal. 44 $111-130(2005)$

21. Lions, JL: Quelques Méthodes de Résolution des Problèmes aux Limites Non Linéaires. Dunod, Paris (1969)

22. Anguiano, M, Caraballo, T, Real, J, Valero, J: Pullback attractors for reaction-diffusion equations in some unbounded domains with an $H^{-1}$-valued non-autonomous forcing term and without uniqueness of solutions. Discret Cont Dyn Syst B. $14,307-326(2010)$

23. Rosa, R: The global attractor for the $2 \mathrm{D}$ Navier-Stokes flow on some unbounded domains. Nonlinear Anal. 32, 71-85 (1998)

24. Temam, R: Navier-Stokes Equations and Nonlinear Functional Analysis. SIAM (series lectures), Philadelphia, 2 (1995)

doi:10.1186/1687-2770-2012-35

Cite this article as: Binh and Anh: Attractors for parabolic equations related to Caffarelli-Kohn-Nirenberg

inequalities. Boundary Value Problems 2012 2012:35.

\section{Submit your manuscript to a SpringerOpen ${ }^{\ominus}$ journal and benefit from:}

Convenient online submission

- Rigorous peer review

- Immediate publication on acceptance

- Open access: articles freely available online

- High visibility within the field

- Retaining the copyright to your article

Submit your next manuscript at $\gg$ springeropen.com 